\title{
The Proliferation of Clinical Practice Guidelines: Professional Development or Medicine-by- Numbers?
}

\author{
Stephen J. Genuis, MD, FRCSC, DABOG
}

In a medical milieu of extensive research, rapidly proliferating information, and a multitude of potential therapies, there has been an escalating trend toward the development and dissemination of clinical practice guidelines outlining investigative and management protocols for clinical problems. There are substantial benefits to providing educational directives and securing widespread adherence to specific clinical practice standards as a means to ensure a consistent acceptable standard-of-care. On the other hand, the increasing tendency to regard authoritative documents as dogma may hinder ongoing medical progress and facilitate the adoption of a "follow-the-recipe" approach to medical practice. A healthy tension between physician autonomy and recommended practice guidelines needs to be cultivated in primary care as well as in specialty clinical practice. In response to increasing concern surrounding issues of impartiality and commercial influence on the development of practice directives, a mechanism designed to assure integrity and credibility of guidelines is required. (J Am Board Fam Pract 2005;18: 419-25.)

The important thing is never to stop questioning. -Albert Einstein

With ever increasing attention given to evidencebased medicine, the proliferation of practice directives in the form of clinical practice guidelines (CPGs) has become a primary mechanism for communicating clinical aspects of emerging therapies and "standard-of-care" expectations to practicing physicians. Recent discussion in the medical literature, however, suggests a need for caution when implementing recommendations contained within CPGs.

The initial spark that ignited my apprehension about practice directives and standard-of-care pronouncements involved the issue of hormone replacement therapy (HRT). When it became fashionable to use HRT containing estrogen and progesterone to prevent osteoporosis, heart disease, and cognitive decline in women, I considered the putative benefits with a somewhat jaundiced eye. During my residency training I had read ex-

Submitted, revised, 21 March 2005.

From the University of Alberta, Edmonton, Alberta, Canada

Conflict of interest: none declared.

Corresponding author: Stephen Genuis, MD, FRCSC, DABOG, 2935 66th Street, Edmonton Alberta, Canada T6K 4C1 (e-mail: sgenuis@ualberta.ca). tensively on the relatively recent diethylstilbestrol (DES) estrogen therapy fiasco, the resultant birth abnormalities, the reproductive dysfunction, and the cancerous sequelae. In an environment of mounting pressure to recommend widespread use of long-term HRT as preventive therapy for healthy women, I reviewed the available information and research. I was astonished to find that within a medical paradigm that emphasizes evidence-based medicine, there was a remarkable paucity of scientific evidence for long-term preventive HRT as the standard-of-care for postmenopausal women. Moreover, I did not understand how HRT had catapulted to become the leading drug sold in America. ${ }^{1}$ A little searching in the library yielded a partial explanation.

A widely read bestseller entitled Everytbing You Wanted to Know about Sex was released in 1969 by the prominent psychiatrist, Dr. David Reuben, setting the stage for the use of long-term HRT. It was his concerted opinion that HRT was an absolute necessity:

"As estrogen is shut off, a woman becomes as close as she can to being a man. Increased facial hair, deepened voice, obesity, and decline of breasts and female genitalia all contribute to a masculine appearance. Not really a man but no longer a functional woman, these individuals 
live in a world of intersex. Having outlived their ovaries, they have outlived their usefulness as human beings." 2

With similar admonitions in books and medical papers from other noted medical professionals, ${ }^{3}$ it is evident why women rushed to consume products that pledged to preclude such disaster and why doctors began to encourage postmenopausal women to take advantage of the medication.

Sifting through the scientific literature, I tried to separate spin from actual facts and data. Short trials of HRT, as well as theoretical calculations and extrapolations from observational studies, were reported. ${ }^{4-8}$ Although information collected by these means can be very important in the innovationdevelopment process, it is low in the "hierarchy of evidence" 9 and thus should be viewed as a starting point for further research, not the basis for widespread change in medical practice. There was a complete dearth of large, well-designed randomized controlled trials confirming that HRT was safe and effective when used as a long-term preventive strategy for healthy postmenopausal patients. ${ }^{10}$ Furthermore, potential risks and uncertainties associated with HRT continued to emerge. ${ }^{11-19}$ Accordingly, I decided not to routinely recommend preventive HRT and I searched for other means to address the health concerns associated with menopause.

Within a medical atmosphere emphasizing evidence-based medicine, however, I puzzled over how the practice patterns of most physicians and the recommendations of respected gynecological associations so quickly evolved into a paradigm where routine HRT was recommended in practice guidelines and had become the "standard-of-care." I found it hard to believe that the medical community would adopt a standard of practice well before there was clear evidence of safety and efficacy. Yet, an exploration of recent medical literature elucidates the details surrounding CPG development and demonstrates why such guidelines and the standard-of-care may not always be the same as "optimal care" for patients.

\section{Clinical Practice Guidelines and the Standard-of-Care}

For the medical profession to maintain credibility as a self-governing entity, it is essential that physicians consistently provide an acceptable level of care and that individual practitioners remain accountable for their management decisions. With this end in mind, however, there has been ever increasing intrusion into physician autonomy and numerous educational efforts designed to ensure widespread adherence to commonly accepted patterns of practice. The standard-of-care, referring to the current acceptable management of a medical problem as determined by consensus among experts, is frequently discussed in educational and legal settings with the connotation that current CPGs relating to a specific health problem represent the contemporary standard-of-care. Although not a technical medical term, the standard-of-care jargon carries the implicit message, however, that alternate management may be substandard care and that deviation from CPGs may be suboptimal management.

Often accompanied by the endorsement of professional organizations and specialty divisions of medicine, CPGs and strategic algorithms, outlining investigative and management paths for clinical problems, have proliferated and currently span several areas of medical practice. These guidelines continue to be produced at an unprecedented rate with well over 1000 CPGs posted on various medical web sites, ${ }^{20-22}$ covering a wide range of topics from osteoporosis to contraception, from atrial fibrillation to Alzheimer disease, and from genetic testing to end-of-life decisions. As well as assisting physicians in clinical practice, standardized guidelines are increasingly used by experts, including lawyers and medical administrators investigating medicolegal and discipline concerns, as a grid by which a doctor's performance is measured and punitive actions are enforced. ${ }^{23,24}$ What is the purpose of CPGs and how are they developed?

CPGs have been succinctly defined as "userfriendly statements that bring together the best external evidence and other knowledge necessary for decision making about a specific health problem." 25 Incorporating the most recent evidence on a given health issue, CPGs are intended to initially define the clinical practice problem, and then present the best evidence pertaining to etiology, prevention, diagnostic investigations, options for therapy with associated risks, anticipated prognosis, and cost-effectiveness data. ${ }^{25-27}$ Furthermore, CPGs should provide options in a manner that can be integrated with clinical experience and the patient's values to effect the best decision in each 
situation. Ideally, the clinician is then encouraged to carefully assess the credibility of each guideline and decide whether the recommendations are useful in his/her practice.

The mechanism for CPG development and the sources of funding may vary. The preferred format for creating a CPG involves the assimilation of intellectual contributions of experienced impartial researchers and clinicians who use the most recent evidence and research on a particular health issue, incorporating the spectrum of available data from the medical and scientific literature. Unbiased information is presented and the strengths, drawbacks, and uncertainties of all recommendations are clarified with every effort made to divorce evidence from opinion. The more common source for CPGs, however, is the publication of the reasoned thoughts of a panel of experts who agree on a specific course of action related to a given health problem; documents fashioned in this way may also be referred to as consensus guidelines. The financial support for the creation of CPGs may originate from public service institutions such as governments or universities; alternatively, the dollars may be provided by vested interests such as industry through direct educational grants or indirectly through funding to persons, professional associations, or illness-specific foundations. Recognized experts whose research or other endeavors are supported by industry are often asked to be part of a task force to prepare CPGs. When perusing CPG publications, however, it is often unclear where the primary source of funding originates and recent analysis reveals that most industry funded CPGs do not disclose the financial affiliation on the published guidelines. ${ }^{28}$

There are well-recognized benefits to providing organized and logical education in the form of CPGs to assist physicians in dealing with patient concerns. With the ongoing plethora of new research and information, combined with the very busy and demanding schedules of most health practitioners, guidelines for specific problems with clear and logical algorithms to approach clinical situations can be an invaluable source of education and a readily available reference. On the other hand, unquestioning acceptance and routine use of such predetermined instructions threatens to reduce medical doctors to the practice of "medicine-bynumbers," with a growing reliance on experts and documents rather than utilization of personal clin- ical judgment. Recent events have prompted a reevaluation of the role of authoritative guidelines as a preferred source for continuing education and up-to-date clinical management strategies.

The medical literature has highlighted mounting concerns related to recommended practice protocols. Many practitioners dealing with individual patients have not fully appreciated, for example, that although CPGs often use broad-based topical headlines such as osteoporosis or asthma, individual patients frequently fall outside the demographics of the specific groups studied to prepare the CPGs. ${ }^{29}$ Frequent examples of this include patients whose specific age group was not researched, patients receiving concomitant therapies, and patients with coexisting medical problems, all groups for whom data may simply not be available. It has also become evident that most CPGs focus heavily on clinical trials and therapeutic interventions, often neglecting etiology, prevention, health promotion, and long-term risks. Furthermore, medical and scientific progress is dependent on "thinking outside the box" with recognition of shortcomings in the status quo; rigid adherence to guidelines may facilitate intellectual laziness and hinder innovation.

Most striking, however, have been concerns related to the mounting number of previously recommended interventions and guidelines, which may ultimately have delivered significant harm. In addition to the HRT example, prior recommendations surrounding the use of potentially dangerous anti-inflammatory therapies ${ }^{30,31}$ are being investigated, and the previous widespread promotion of antidepressant drugs which "may worsen depression or trigger suicidal or aggressive impulses" ${ }^{32}$ in youth, is also being assessed. Such revelations beg the question of how recommended guidelines that have enormous potential for harm can exist in an allegedly scientific atmosphere of erudite experts and meticulous, systematic evidence-based scientific research.

\section{Vested Interests and Medicine}

The basic foundation of medical education and clinical practice is research studies published in the medical and scientific literature. The information contained within these publications is supposedly based on objective evidence and rigorous adherence to scientific principles and methodology. The scientific data, which is subsequently relayed to physicians at medical education events and through 
publications, is required for competent medical practice and continued progress in health care. Evidence-based data supports or negates the use of medical interventions and innovative studies form the building blocks for subsequent exploration and research. It is from such sources that the CPGs and the standard-of-care concepts are developed.

Recent evidence presented by various medical editors and reporters, however, exposes concerning information about medical research and education. Many articles indicate that commercial interests provide significant funding to, and exert influence on the following: academic medical institutions, clinical research, scientists who do the research, publications that report research, and physicians who teach and report research to the medical community. ${ }^{33-35}$ Many of the current CPGs and Consensus Conference Statements, documents which are often interpreted by practicing MDs as being authoritative with regard to patient management, are being funded by industry through grants to persons and to illness-specific foundations. ${ }^{36}$ In fact, a recent study reported in the fournal of the American Medical Association revealed that $87 \%$ of authors of CPGs had some form of interaction with industry. ${ }^{28}$ The net result is that CPGs, created to influence the practice behaviors of vast numbers of physicians, ${ }^{28}$ are often used as ingenious marketing tools. A brief saunter into the recent literature discussing the influence of industry on medicine is telling.

Academic institutions, frequently regarded as the bastions of learning, innovative thought, and free speech, are significantly impacted by the influence of industry. Two former principal editors of the New England Fournal of Medicine (NEFM) recently concluded that academic institutions are increasingly being tainted by commercial interests. ${ }^{33,37}$ They claim that "virtually every researchintensive medical center in the country now has contractual ties with one or more drug firms" and that "in many medical specialties these days, it is almost impossible to find an expert who is not receiving payments from one or more drug companies in the field." 33

In addition, concern has recently been expressed that companies are involved in research design and have the power to influence study results. ${ }^{34,38,39}$ There is recurring data demonstrating a link between industry-backed research and positive conclusions of clinical trials. In fact, specific drugs being researched are recommended as the treatment of choice 3 times more often if the research is funded by a for-profit versus a nonprofit organization. ${ }^{40}$ Researchers supported by industry are often required to sign confidentiality agreements or "gag clauses" limiting their freedom to disseminate information regarding ineffective or dangerous outcomes. $^{12,41}$

The integrity of medical reporting has also become an issue. ${ }^{12}$ Scientific authors are often supported by companies whose products they are researching. For example, a recent study revealed that $96 \%$ of those who wrote articles affirming the value of specific cardiac medications had a financial relationship with the manufacturer. ${ }^{42}$ A significant proportion of scientific articles appearing in major medical journals under the supposed authorship of scientists and doctors have actually been written by ghostwriters on the payroll of industry. ${ }^{12,43}$ Some experts whose names appear on articles are compensated for "lending" their names (and reputations) but have had nothing to do with either the research or crafting of the article itself. In 2003, for example, the $N E 7 M$ retracted a published article, which was supposedly penned by doctors from the National Heart Institute, when one of the listed authors told the editor he had never seen any version of the manuscript. ${ }^{43}$ Reflecting on concerns related to industry influence on medical publications, Dr. Richard Horton, editor of the prestigious Lancet, recently stated that "some medical journals ... have devolved into information-laundering operations for the pharmaceutical industry." 44

Dissemination of health information to physicians, the last step in the educational process regarding practice guidelines, is a high priority for industry. In presentations at various educational venues, corporate sponsors often support "thought leaders," influential specialists or experts in a particular subject area, to educate practitioners about recent research, recommended guidelines, and the standard-of-care. Industry-funded "throwaway" medical magazines, look-alike pseudojournals and other questionable publications arrive routinely in physicians' mailboxes and keep recommended treatment protocols and product names foremost in MDs minds. ${ }^{12,45}$

Many physicians and the general public are not fully cognizant of the potential impact of economics on medical expertise. Authorities or experts in the medical realm are often perceived as a group of 
objective professionals who set out to provide the public and health communities with reliable untainted up-to-date health information. It is evident, however, that many experts who advise federal regulators, travel the globe encouraging the use of various therapies, sit on editorial boards for major journals, and write scientific publications may frequently be in a conflict of interest position, and their recommendations may, at times, be subjective. For example, the recent revelation that hundreds of government scientists at the National Institutes of Health have taken fees, stock, or stock options from biomedical companies over the past 5 years with no requirement to publicly acknowledge a potential conflict of interest is of concern. ${ }^{46,47}$ When such scientists and experts are the intellectual source for the development of CPGs, there may be cause for questions about objectivity.

Recognizing that the development and dissemination of medical care guidelines may be influenced by factors other than the well-being of patients, ethical angst may arise for individual physicians when a given standard-of-care or CPG does not seem to be in accordance with their clinical acumen, their assessment of credible information, and ultimately what they believe is best for their patient. Is it acceptable to incorporate clinical experience and appraise the scientific literature, come to a different conclusion, and practice conscientiously in a way that may not coincide exactly with the prescribed standard-of-care and CPGs?

\section{Recommendations}

It is hard to overstate the impact when a practitioner hears that a specific therapy is the standard-ofcare. Messages communicated by thought-leaders have a profound impact on the practice patterns of physicians. ${ }^{48}$ Although not explicitly stated, such practice guidelines and directives implicitly translate into practice imperatives when considering medicolegal and possible discipline sequelae. This paradigm needs to be re-evaluated.

During medical school, I was taught that "the physician-patient relationship is based on a patient's trust that the physician is committed to the patient's best interest." ${ }^{49}$ Doctors should make clinical decisions integrating the physician's best clinical judgment with the patient's needs and values, regardless of any practice directive. A CPG recommendation that is contrary to the way a person practices, however, should serve as an impetus to re-evaluate and reassess patterns of practice. MDs should seek advice from trusted sources before changing established practice patterns and to support their medical decisions, doctors should have a reasonable, well thought out argument and be prepared to intelligently defend their choices. Many times in history, including the recent HRT example, existing medical dogma has been proven entirely wrong with the passage of time; accordingly, it should be permissible for practitioners to pursue credible scientific fact as the ultimate authority, rather than simply accepting expert panels or CPGs as the ultimate source of credible scientific fact.

The issue of legal liability and discipline against physicians not adhering to CPGs or the standardof-care raises important ethical questions and needs to be discussed and explored in the medical community. Adhering to a practice guideline that a practitioner feels is suboptimal, however, is questionable ethically-acting in a fashion contrary to what a practitioner determines to be in the patient's best interests may compromise the relationship and diminish professional integrity. In the face of dubious clinical directives, most patients still want a doctor who would not compromise what he/she thinks is best and who refuses to conform to practicing medicine in ways he/she perceives to be inappropriate.

Physicians should scrutinize new medical information as well as the persons presenting such information; evaluating and integrating research into clinical practice is a vital lifelong skill for practitioners. ${ }^{50}$ With voluminous amounts of ongoing research and thousands of articles published each month, with emerging new subspecialty areas of medicine, and with all the bioethical and administrative dimensions to contemporary medical practice, however, it is very difficult practically for primary practitioners to remain current on everything that is happening in clinical medicine. Furthermore, most physicians are busy providing necessary care and service to needy patients and do not always have time available to study numerous medical journals to discern what is biostatistically relevant. Accordingly, reliable medical education through credible CPGs developed by trustworthy experts can be invaluable. Yet, how can the development of credible CPGs be secured?

An international registry with exacting criteria for CPGs may be required and nonprofit institu- 
tions motivated by public interest need to be involved in the development of medical practice directives. A well-developed "official" set of CPGs should be explicit, transparent, and publicly accountable in its methods with established criteria to avoid conflict of interest. In the development and production of guidelines, existing published research should be scrutinized for the possibility of partiality or bias and every effort should be made to eliminate conflict of interest and the impact of commercialism. Regular re-evaluation and updates of CPGs should be included in the process and easy access to approved guidelines should be secured.

\section{Conclusion}

To pursue optimal care for patients, the medical profession must increasingly seek to ensure that CPGs, the standard-of-care, and medical education at all stages be directed at benefiting health, untainted by the influences of commercialization. Although much of what is found in the medical literature is credible and the enormous contribution of ethical industry and principled pharmaceutical professionals is invaluable, current reality demands that physicians learn to discern fact from sales pitch. When the Women's Health Initiative and numerous other studies demonstrated that HRT significantly endangers the long-term health and well-being of women, ${ }^{18,51-53}$ the shortcomings of a standard-of-care approach to management decisions became clearly evident. Although a mechanism is required to re-evaluate the mounting plethora of existing CPGs and to secure credible guidelines as a resource for cutting edge information, good clinical medicine entails a healthy tension between physician autonomy and recommended patterns of practice.

\section{References}

1. Meyer VF. The medicalization of menopause: critique and consequences. Int J Health Services 2001; 31:769-92.

2. Reuben D. Everything you always wanted to know about sex. New York: David McKay Company, Inc., 1969.

3. Wilson RA. Feminine forever. New York: M. Evans and Company, 1966.

4. Grodstein F, Manson JE, Colditz GA, Willett WC, Speizer FE, Stampfer MJ. A prospective, observational study of postmenopausal hormone therapy and primary prevention of cardiovascular disease. Ann Intern Med 2000;133:933-41.
5. The Writing Group for the PEPI Trial. Effects of estrogen or estrogen/progestin regimens on heart disease risk factors in postmenopausal women. The Postmenopausal Estrogen/Progestin Interventions (PEPI) Trial. JAMA 1995;273:199-208.

6. Col NF, Eckman MH, Karas RH, et al. Patientspecific decisions about hormone replacement therapy in postmenopausal women. JAMA 1997;277: $1140-7$.

7. Stefanick ML. Estrogen, progestogens and cardiovascular risk. J Reprod Med 1999;44:221-6.

8. Paganini-Hill A, Henderson VW. Estrogen deficiency and risk of Alzheimer's disease in women. Am J Epidemiol 1994;140:256-61.

9. Greenhalgh T. How to read a paper. Getting your bearings (deciding what the paper is about). BMJ 1997;315:243-6.

10. Yusuf S, Anand S. Hormone replacement therapy: a time for pause. CMAJ 2002;167:357-9.

11. Bergkvist L, Adami HO, Persson I, Hoover R, Schairer C. The risk of breast cancer after estrogen and estrogen-progestin replacement. N Engl J Med 1989;321:293-7.

12. National Women's Health Network. The truth about hormone replacement therapy. Roseville (CA): Prima Publishing; 2002.

13. Troisi RJ, Speizer FE, Willett WC, Trichopoulos D, Rosner B. Menopause, postmenopausal estrogen preparations, and the risk of adult-onset asthma: a prospective cohort study. Am J Respir Crit Care Med 1995;152:1183-8.

14. Lankish PG, Droge M, Gottesleben F. Drug induced acute pancreatitis: incidence and severity. Gut 1995;37:565-7.

15. LeResche L, Saunders K, Von Korff MR, Barlow W, Dworkin SF. Use of exogenous hormones and risk of temporomandibular disorder pain. Pain 1997;69: 153-60.

16. Meier CR, Sturkenboom MC, Cohen AS, Jick H. Postmenopausal estrogen replacement therapy and the risk of developing systemic lupus erythematosus or discoid lupus. J Rheumatol 1998;25:1515-9.

17. Mitka M. New advice for women patients about hormone therapy and the heart. JAMA 2001;286: 907.

18. Chen CL, Weiss NS, Newcomb P, Barlow W, White E. Hormone replacement therapy in relation to breast cancer. JAMA 2002;287:734-41.

19. Mamdani M, Tu K, van Walraven C, Austin PC, Naylor CD. Postmenopausal estrogen replacement and increased rates of cholecystectomy and appendectomy. CMAJ 2000;162:1421-4.

20. National Guideline Clearinghouse 2005. [accessed 4 February 2005]. Available at: bttp://www.guideline. gov/.

21. Primary Care Clinical Practice Guidelines 2005. [accessed 4 February 2005]. Available at: bttp:// medicine.ucsf.edu/resources/guidelines/. 
22. Canadian Medical Association Clinical Practice Guidelines 2005. [accessed 4 February 2005]. Available at: http://mdm.ca/cpgsnew/cpgs/index.asp.

23. Hurwitz B. How does evidence based guidance influence determinations of medical negligence?. BMJ 2004;329:1024-8.

24. Keffer JH. Guidelines and algorithms: perceptions of why and when they are successful and how to improve them. Clin Chem 2001;47:1563-72.

25. Sackett DL, Richardson WS, Rosenberg W, Haynes RB. Evidence-based medicine: how to practice and teach EBM. New York: Churchill Livingstone; 1997.

26. Hayward RS, Wilson MC, Tunis SR, Bass EB, Guyatt G. Users' guides to the medical literature. VIII. How to use clinical practice guidelines. A. Are the recommendations valid? The Evidence-Based Medicine Working Group. JAMA 1995;274:570-4.

27. Wilson MC, Hayward RS, Tunis SR, Bass EB, Guyatt G. Users' guides to the Medical Literature. VIII. How to use clinical practice guidelines. B. What are the recommendations and will they help you in caring for your patients? The Evidence-Based Medicine Working Group. JAMA 1995;274:1630-2.

28. Choudhry NK, Stelfox HT, Detsky AS. Relationships between authors of clinical practice guidelines and the pharmaceutical industry. JAMA 2002;287: 612-7.

29. Julian DG. What is right and what is wrong about evidence-based medicine?. J Cardiovasc Electrophysiol 2003;14:S2-5.

30. Topol EJ. Failing the public health-rofecoxib, Merck, and the FDA. N Engl J Med 2004;351: 1707-9.

31. Fitzgerald GA. Coxibs and cardiovascular disease. N Engl J Med 2004;351:1709-11.

32. Kondro W. UK bans, Health Canada warns about antidepressants. CMAJ 2004;171:23.

33. Relman AS, Angell M. America's other drug problem: how the drug industry distorts medicine and politics. New Republic 2002;587:27-41.

34. Angell $M$. Is academic medicine for sale?. N Engl J Med 2000;342:1516-8.

35. Moynihan R. Who pays for the pizza? Redefining the relationships between doctors and drug companies. 1: Entanglement. BMJ 2003;326:1189-92.

36. CMAJ Editorial. Obituary: the Canadian task force on preventive health care. CMAJ 2003;169:1137.

37. Angell $M$. The truth about the drug companies: how they deceive us and what to do about it. New York: Random House; 2004.
38. Bodenheimer T. Uneasy alliance-clinical investigators and the pharmaceutical industry. N Engl J Med 2000;342:1539-44.

39. Bero LA, Rennie D. Influences on the quality of published drug studies. Int J Technol Assess Health Care 1996;12:209-37.

40. Lexchin J, Bero LA, Djulbegovic B, Clark O. Pharmaceutical industry sponsorship and research outcome and quality: systematic review. BMJ 2003;326: 1167-70.

41. Dehaas D. Much ado about nothing: whistle-blower or busybody? MD Canada 2003;1:6, 19-30.

42. Stelfox HT, Chua G, O'Rourke K, Detsky AS. Conflict of interest in the debate over calcium-channel antagonists. N Engl J Med 1998;338:101-6.

43. Barnett A. Revealed: how drug firms 'hoodwink' medical journals. Pharmaceutical giants hire ghostwriters to produce articles - then put doctors' names on them. The Observer. 2003 Dec 7.

44. Horton R. MMR: science and fiction. London: Granta Books; 2004.

45. Rochon PA, Bero LA, Bay AM, et al. Comparison of review articles published in peer-reviewed and throwaway journals. JAMA 2002;287:2853-6.

46. Harvard School of Public Health: World Health News. Willman D. The National Institutes of Health: Public servant or private marketer? Los Angeles Times. 2004 Dec 22.

47. Willman D. Stealth merger: drug companies and government medical research. Los Angeles Times. 2003 Dec 7.

48. Rogers EM. Diffusion of Innovations. New York: The Free Press, 1995.

49. Moschovis PP. When cultures are wrong. JAMA 2002;288:1131-2.

50. Haines A, Donald A. Getting research findings into practice: making better use of research findings. BMJ 1998;317:72-5.

51. Shumaker SA, Legault C, Rapp SR, et al. Estrogen plus progestin and the incidence of dementia and mild cognitive impairment in postmenopausal women: the Women's Health Initiative Memory Study: a randomized controlled trial. JAMA 2003;289:265162.

52. Herrington DM, Howard TD. From presumed benefit to potential harm-hormone therapy and heart disease. N Engl J Med 2003;349:519-21.

53. Wassertheil-Smoller S, Hendrix SL, Limacher M, et al. Effect of estrogen plus progestin on stroke in postmenopausal women: the Women's Health Initiative: a randomized trial. JAMA 2003;289:2673-84. 